\title{
Peertechz
}

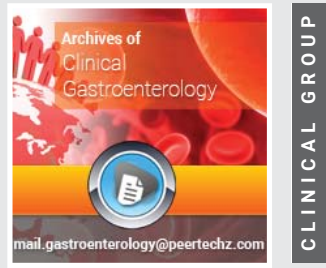

\section{Short-term outcomes of}

\section{laparotomy in the two teaching} hospitals of gulu university, northern uganda

\section{Amone $\mathrm{D}^{1}$, Okello $\mathrm{TR}^{1 *}$, Okot $\mathrm{C}^{2}$, Kitara $\mathrm{DL}^{3}$, Mugabi $\mathrm{P}^{3}$ and Ogwang $\mathrm{DM}^{4}$}

1Department of Surgery, Graduate Trainee and Assistant lecturer in Surgery and Anatomy, Gulu

University, Senior Lecturer and Head Lira University, Uganda

${ }^{2}$ Professor and Head Department of Surgery, Medical officer Special Grade, Gulu Regional Referral

Hospital and Associates. Gulu University, Uganda

${ }^{3}$ Lecturer, Department of Surgery, Gulu University, Uganda

${ }^{4}$ Senior Consultant Surgeon and Institutional Director St. Mary's Hospital Lacor, Uganda
Received: 15 October, 2020

Accepted: 04 November, 2020

Published: 17 November, 2020

*Corresponding author: Tom Richard Okello, Senior Lecturer and Head of Surgery Department, lira University, Uganda, Tel: +256772473510; Email: okellotomrich@gmail.com

Keywords: Laparotomy; Short-term outcome of surgery; Peritonitis; Surgical site infections: Complication of laparotomy

https://www.peertechz.com

\section{Check for updates}

\section{Abstract}

Purpose: The purpose of this study was to examine the clinical indication for laparotomy, the intra-operative findings and the 30 days post-operative outcome of laparotomy in Gulu university teaching hospitals.

Methods: Using an approved protocol, a six month descriptive longitudinal study was conducted on patients undergoing laparotomy in the two main Gulu University teaching hospitals of St. Mary's Hospital Lacor and Gulu regional referral. Using a sample size of 66 , cases were recruited consecutively, clerked, investigated and conventionally prepared for surgery. Intra-operative diagnosis was ascertained as well as the operative procedure and post-operatively the patients were followed up for 30 days complications including death.

Results: Overall, the mean age was 35.04yrs (SD+/- 16.522), but there were more males (59.4\%) than females (40.6\%). There was a statistically significant positive correlation between the clinical diagnosis and the intraoperative findings $(r=0.405, P$ value $=0.001)$. Within the 30 days, the most frequent complication observed was surgical site infection (SSI) $(20 \%, n=13)$, followed by wound dehiscence $(17.2 \%, n=11)$, crude mortality rate was $15.6 \%$, ( $n=10)$ and complication requiring emergency reoperation $(10.9 \%, n=7)$. Ileal perforations tended to have bad outcomes. Age of patient was found to be a significant factor in determining the outcome.

Conclusion: Descriptive longitudinal study on both elective and emergency laparotomy is possible in our setting. Whereas patients' age is an important factors in determining outcome and ileal perforation tend to do better if prioritized with ileostomy, overall the 30 days mortality rate for laparotomy was $15.6 \%$.

\section{Introduction}

The word laparotomy is derived from the Greek words lapara, meaning flank, and tomy, meaning cut. In surgical practice, this translates to a big cut in the abdomen to gain access to the peritoneal cavity often midline along the linea alba [1]. Laparotomy is therefore a surgical incision into the abdominal cavity [2]. Laparotomies are the most common operations performed in many hospitals for both emergency and elective conditions for example intra-abdominal infections, bowel obstructions, tumors, hernias and abdominal trauma [3]. In the United Kingdom, this is a common procedure with approximately 30,000 to 50,000 performed annually [1]. Broadly, its indications could be divided into acute abdomen and trauma but of the acute abdomens, $57 \%$ are due to gastrointestinal perforation, $33 \%$ have intestinal obstruction and in trauma $63 \%$ have blunt abdominal trauma and the rest penetrating injury [4]. Kakande, et al. (2001) reported that 
intestinal obstruction represent the commonest indication for laparotomies [5]. Other authors however report that trauma associated with hemodynamic instability tops amongst the indications $[6,7]$.

However in the Post-laparatomy period, some patients recover and are discharged uneventfully whereas others develop complications which can lead to prolonged hospital stay, morbidity and mortality $[3,8]$. According to Tengberg, et al. (2016), major complication occur in $47 \%$ of all laparatomy patients within 30 days of the surgery out of which the unadjusted 30 day mortality accounts for, $20.2 \%$ [9]. Another study found that nausea \& vomiting account for (56\%) post laparotomy outcome, followed by chest infection(38\%), wound complications $(33 \%)$ and paralytic ileus $(26 \%)$ to name but a few [10].

Rationale: Many patients undergo laparotomy but its outcome in not well documented in our setting. Indeed limited data is have been published describing the indications, postoperative course and the temporal pattern of complications after laparotomy, thus this study.

\section{Objective}

1. To examine the extent of clinical indication for laparotomy compared to the intra-operative findings

2. The Examine the 3odays post-operative outcome of laparotomy in Gulu university teaching hospitals.

\section{Methods}

Using an approved protocol, a descriptive longitudinal study was conducted on patients undergoing laparotomy between $1^{\text {st }}$ July 2017 and $31^{\text {st }}$ December 2017 in the two Gulu University teaching hospital of Gulu Regional Referral Hospital and St Mary's Hospital, Lacor. Gulu regional Referral Hospital is a 450 bed government hospital, found in the middle of Gulu City while St Mary's Hospital Lacor is a 482 bed and a faith based Not for Profit hospital located about $5 \mathrm{Km}$ from Gulu City center along Juba road.

Patients presented to these hospitals either as emergency or elective, they were clerked and examined in the conventional way and a diagnosis reached. Baseline investigation like complete blood count (CBC ultrasonograhy (US), plain x-ray was done and when needed a senior surgeon was consulted on the way forward. Patients whose diagnosis needed a laparotomy intervention were then prepared in the routine way and after their consent for the surgery, the research assistant approached them for informed consent to enrolled into the study. Amongst those enrolled in the study, data was then collected consecutively by double blinded but trained research assistants (medical officers). The sample size was 66 participants determined by Kish, Leslie. 1965 formula.

The quantitative data collection instrument used was approved by the local research ethics committee and consisted of a coded semi-structured interviewer administered questionnaire designed to allow post-operative follow-up of the study participants up to 30 days or day of death. The following variables were collected, entered and analysed using SPSS version20: biographic information, clinical diagnosis (indication for laparotomy including relevant investigation), intra-operative findings and the major outcome like recovery or death, wound dehiscence, Surgical Site infection, duration hospitalization, post-operative peritonitis, leakage, relaparotomy and organ failure as outcome measures. The result was the presented in the tables below.

\section{Results}

A total of 66 patients participated in the study out of whom only $64(97 \%)$ had complete data for analysis. All the participants underwent laparotomy operation either as elective or emergency between $1^{\text {st }}$ July 2017 and $31^{\text {st }}$ December 2017.The majority of whom were peasant farmers $(58 \%, \mathrm{~N}=64)$, followed by students $(16 \%, \mathrm{~N}=64)$, civil servants $(4.7 \%, \mathrm{~N}=64)$ and drivers, car mechanic, security guards each accounted for $3.1 \%$ respectively (Chi-square 222.524, $\mathrm{P}$ value 0.000 ). Furthermore there were more males than female.

Age and sex distribution: As shown in Table 1, the majority of patients who underwent laparotomy procedure in our setting were the youth of $19-35 \mathrm{yrs}(\mathrm{N}=64, \mathrm{n}=23,36 \%)$ and Adults of 36-65yrs $(\mathrm{N}=64, \mathrm{n}=22,34.4 \%)$. Overall, the mean age was 35.04yrs (SD+/- 16.522). However, there were more males $(59.4 \%)$ than females $(40.6 \%)$ who underwent laparotomy with male: female ratio of 1.5:1 respectively. Except for children within the age groups of $10-18 \mathrm{yrs}$, there was a tendency of male gender predominance amongst those who were operated though the difference was not statistically significant (ChiSquare 3.265 , $\mathrm{P}$ value $>0.05$ )

\begin{tabular}{|c|c|c|c|}
\hline Table 1: Age Sex Distribution. & \multicolumn{3}{|c|}{ Gender of Participant } \\
\cline { 2 - 4 } Age group of patients & Male & Female & Total \\
\hline Child (10-18yrs) & $4(40 \%)$ & $6(60 \%)$ & $10(15.6 \%)$ \\
\hline Youth (19-35yrs) & $15(65.2 \%)$ & $8(34.8 \%)$ & $23(35.9 \%)$ \\
\hline Adult (36-65yrs) & $13(59.1 \%)$ & $9(40.9 \%)$ & $22(34.3 \%)$ \\
\hline Elderly (>65yrs) & $2(100 \%)$ & 0 & $2(3.1 \%)$ \\
\hline Others (<10yrs) & $4(57.1 \%)$ & $3(42.9 \%)$ & $7(11 \%)$ \\
\hline Total & $38(59.4 \%)$ & $26(40.6 \%)$ & $64(100 \%)$ \\
\hline
\end{tabular}

Clinical diagnosis (indication) and Intraoperative Finding: Table 2 shows a comparison between clinical indication for laparotomy and the intra-operative findings. Whereas the commonest clinical indication for laparotomy in this study was found to be peritonitis $(36 \%, N=64, n=23)$ followed by intestinal obstruction $(23.4 \%, \mathrm{~N}=64, \mathrm{n}=15)$ and gut perforation, Appendicitis, penetrating abdominal injury each accounting for $7.8 \%$ respectively, the intra-operative finding majorly were peritonitis $(17.1 \%)$ of which perforated peptic ulcer (PUD) accounted for $6.3 \%(n=4)$, perforation of ileum $(n=7,10.9 \%)$ followed by intestinal obstruction due to adhesions $(n=8$, $12.5 \%)$ and Appendicitis ( $\mathrm{n}=5,7.8 \%)$.

Furthermore from Table 2, out of the $n=23$ patients who presented with clinical signs and symptoms of peritonitis, $26.1 \%$ were found to have perforated of ileum and perforated PUD accounted for $17.4 \%$. The majority of patients who presented 
Table 2: Correlation of Clinical Diagnosis and the Intra Operative Findings.

\begin{tabular}{|c|c|c|c|c|c|c|c|c|c|c|c|c|c|c|}
\hline \multirow[b]{2}{*}{$\begin{array}{l}\text { Intra-operative } \\
\text { finding }\end{array}$} & \multicolumn{14}{|c|}{ Clinical Diagnosis } \\
\hline & Peritonitis & $\begin{array}{l}\text { Liver } \\
\text { injury }\end{array}$ & $\begin{array}{c}\text { Intestinal } \\
\text { Obstruction }\end{array}$ & $\begin{array}{c}\text { Paralytic } \\
\text { Ileus }\end{array}$ & \begin{tabular}{|c|} 
Gut \\
perfora- \\
tion
\end{tabular} & $\begin{array}{c}\text { Penetrating } \\
\text { Abdominal } \\
\text { injury }\end{array}$ & $\begin{array}{c}\text { Appendi- } \\
\text { sitis }\end{array}$ & $\begin{array}{c}\text { Cancer } \\
\text { colon }\end{array}$ & $\begin{array}{c}\text { Intussuc- } \\
\text { eption }\end{array}$ & \begin{tabular}{|c|} 
Blunt \\
abdominal \\
Trauma
\end{tabular} & $\begin{array}{l}\text { CA head } \\
\text { Pancreas }\end{array}$ & $\begin{array}{c}\text { Abdominal } \\
\text { Abscess }\end{array}$ & $\begin{array}{c}\text { FB } \\
\text { Stomach }\end{array}$ & Total \\
\hline $\begin{array}{l}\text { Perforated } \\
\text { ileum }\end{array}$ & $6(26.1 \%)$ & 0 & $1(6.7 \%)$ & 0 & 0 & 0 & 0 & 0 & 0 & 0 & 0 & 0 & 0 & $7(10.9 \%)$ \\
\hline Perforated PUD & $4(17.4 \%)$ & 0 & 0 & 0 & 0 & 0 & 0 & 0 & 0 & 0 & 0 & 0 & 0 & $4(6.3 \%)$ \\
\hline $\begin{array}{l}\text { Perforated } \\
\text { jujenum }\end{array}$ & $1(4.3 \%)$ & 0 & 0 & 0 & 0 & 0 & 0 & 0 & 0 & 0 & 0 & 0 & 0 & $1(1.6 \%)$ \\
\hline Liver Laceration & 0 & $\begin{array}{c}1 \\
(100 \%)\end{array}$ & 0 & 0 & 0 & 0 & 0 & 0 & 0 & $1(33.3 \%)$ & 0 & 0 & 0 & $2(3.1)$ \\
\hline Appenditis & $1(4.3)$ & 0 & $2(13.3 \%)$ & $1(100 \%)$ & 0 & 0 & 0 & $1(50 \%)$ & 0 & 0 & 0 & 0 & 0 & $5(7.8 \%)$ \\
\hline $\begin{array}{l}\text { Intestinal } \\
\text { Gangrene }\end{array}$ & 0 & 0 & $1(6.7 \%)$ & 0 & $2(40 \%)$ & 0 & 0 & 0 & $1(100 \%)$ & 0 & 0 & 0 & 0 & $4(6.3 \%)$ \\
\hline $\begin{array}{l}\text { Normal } \\
\text { abdomen }\end{array}$ & $3(13 \%)$ & 0 & 0 & 0 & 0 & $2(40)$ & 0 & 0 & 0 & 0 & 0 & 0 & 0 & $5(7.8 \%)$ \\
\hline Cancer colon & 0 & 0 & 0 & 0 & 0 & 0 & $1(20 \%)$ & 0 & 0 & 0 & 0 & 0 & 0 & $1(1.6 \%)$ \\
\hline $\begin{array}{l}\text { Compound } \\
\text { volvulus }\end{array}$ & $2(8.7 \%)$ & 0 & $1(6.7 \%)$ & 0 & 0 & 0 & 0 & 0 & 0 & 0 & 0 & 0 & 0 & $3(4.7 \%)$ \\
\hline $\begin{array}{c}\text { Leaking } \\
\text { Anastomosis }\end{array}$ & $1(4.3 \%)$ & 0 & 0 & 0 & 0 & 0 & 0 & 0 & 0 & 0 & 0 & 0 & 0 & $1(1.6 \%)$ \\
\hline $\begin{array}{l}\text { Redundant } \\
\text { Sigmoid colon }\end{array}$ & 0 & 0 & 0 & 0 & 0 & 0 & 0 & $1(50 \%)$ & 0 & 0 & 0 & 0 & 0 & $1(1.6 \%)$ \\
\hline Adhesions & $2(8.7 \%)$ & 0 & 5 (33.3\%) & 0 & 0 & 0 & 0 & 0 & 0 & 0 & 0 & $1(100 \%)$ & 0 & $8(12.5 \%)$ \\
\hline $\begin{array}{l}\text { Perforated } \\
\text { Stomach }\end{array}$ & 0 & 0 & 0 & 0 & $1(20 \%)$ & $1(20 \%)$ & 0 & 0 & 0 & 0 & 0 & 0 & 0 & $2(3.1 \%)$ \\
\hline $\begin{array}{l}\text { Ileo-ileal } \\
\text { knotting }\end{array}$ & 0 & 0 & $1(6.7 \%)$ & 0 & 0 & 0 & $1(20 \% 0$ & 0 & 0 & 0 & 0 & 0 & 0 & $2(3.1 \%)$ \\
\hline Terminal ileitis & $1(4.3 \%)$ & 0 & 0 & 0 & 0 & 0 & $1(20 \% 0$ & 0 & 0 & 0 & 0 & 0 & 0 & $2(3.1 \%)$ \\
\hline $\begin{array}{l}\text { Ruptured } \\
\text { Kidney }\end{array}$ & 0 & 0 & 0 & 0 & 0 & 0 & 0 & 0 & 0 & $1(33.3 \%)$ & 0 & 0 & 0 & $1(1.6 \%)$ \\
\hline Sigmoid Volulus & 0 & 0 & $1(6.7 \%)$ & 0 & $1(20 \%)$ & 0 & 0 & 0 & 0 & 0 & 0 & 0 & 0 & $2(3.1 \%)$ \\
\hline $\begin{array}{l}\text { Perforated } \\
\text { Duodenum }\end{array}$ & 0 & 0 & 0 & 0 & $1(20 \%)$ & 0 & 0 & 0 & 0 & 0 & 0 & 0 & 0 & $1(1.6 \%)$ \\
\hline Intussuception & 0 & 0 & $2(13.3 \%)$ & 0 & 0 & 0 & 0 & 0 & 0 & 0 & 0 & 0 & 0 & $2(3.1 \%)$ \\
\hline Cecal mass & 0 & 0 & 0 & 0 & 0 & 0 & $1(20 \%)$ & 0 & 0 & 0 & 0 & 0 & 0 & $1(1.6 \%)$ \\
\hline $\begin{array}{c}\text { Cancer } \\
\text { Pancreas }\end{array}$ & 0 & 0 & 0 & 0 & 0 & 0 & 0 & 0 & 0 & 0 & $1(100 \%)$ & 0 & 0 & $1(1.6 \%)$ \\
\hline $\begin{array}{l}\text { Ruputred } \\
\text { Spleen }\end{array}$ & 0 & 0 & 0 & 0 & 0 & 0 & 0 & 0 & 0 & $1(33.3 \%)$ & 0 & 0 & 0 & $1(1.6 \%)$ \\
\hline $\begin{array}{c}\text { Messenteric } \\
\text { Adenitis }\end{array}$ & $1(4.3 \%)$ & 0 & 0 & 0 & 0 & 0 & 0 & 0 & 0 & 0 & 0 & 0 & 0 & $1(1.6 \%)$ \\
\hline $\begin{array}{l}\text { Perforated } \\
\text { Cecum }\end{array}$ & 0 & 0 & 0 & 0 & 0 & $2(40 \%)$ & 0 & 0 & 0 & 0 & 0 & 0 & 0 & $2(3.1 \%)$ \\
\hline $\begin{array}{l}\text { Abdominal } \\
\text { Abscess }\end{array}$ & 0 & 0 & $1(6.7 \%)$ & 0 & 0 & 0 & 0 & 0 & 0 & 0 & 0 & 0 & 0 & $1(1.6 \%)$ \\
\hline FB Stomach & 0 & 0 & 0 & 0 & 0 & 0 & 0 & 0 & 0 & 0 & 0 & 0 & $1(100 \%)$ & $191.6 \% 0$ \\
\hline $\begin{array}{l}\text { Perforated } \\
\text { Appendix }\end{array}$ & $1(4.3 \%)$ & 0 & 0 & 0 & 0 & 0 & $1(20 \%)$ & 0 & 0 & 0 & 0 & 0 & 0 & $2(3.1 \%)$ \\
\hline Total & 23 & 1 & 15 & 1 & 5 & 5 & 5 & 2 & 1 & 3 & 1 & 1 & 1 & $64(100 \%)$ \\
\hline
\end{tabular}

with features of intestinal obstruction were found to have adhesion (33.3\%), followed by Intussusception, appendicitis (13.3\%) and ileal perforation, abdominal abscess and sigmoid volvulus (each accounted for $6.7 \%$ respectively). Overall negative laparotomy findings were $13 \%$, compound volvulus were $8.7 \%$, Intestinal adhesion were $8.7 \%$ and terminal ileitis and mesenteric adenitis each accounted for $4.3 \%$ respectively. Overall there was a statistically significant positive correlation between the clinical diagnosis and the intraoperative findings $(\mathrm{r}=0.405, \mathrm{P}$ value $=0.001)$.
Operative procedures: Table 3, shows the various operative procedures offered to patients who underwent laparotomy within the study period. Most of the surgeries were performed by medical officers but consultants were always on standby whenever required. The medical officers consisted of surgical residents and pre-residency medical doctor attached in the department of surgery. As shown in Table 3, a variety of surgical procedures were performed depending on the intra-operative finding. Remarkably of the patients whose intraoperative finding was adhesion $(\mathrm{n}=8), 62.5 \%$ were operated adhesiolysis, 
Table 3: Operative procedure.

\begin{tabular}{|c|c|c|c|c|c|c|c|c|c|c|c|c|c|c|c|c|c|}
\hline \multirow{2}{*}{\begin{tabular}{|c|} 
\\
Intra- \\
operative \\
finding \\
\end{tabular}} & \multicolumn{2}{|c|}{$\begin{array}{l}\text { Operative } \\
\text { Procedure }\end{array}$} & \multirow[b]{2}{*}{$\begin{array}{l}\text { Adhesio- } \\
\text { lysis }\end{array}$} & \multirow[b]{2}{*}{ Repair } & \multirow[b]{2}{*}{$\begin{array}{l}\text { Append- } \\
\text { ectomy }\end{array}$} & \multirow[b]{2}{*}{$\begin{array}{l}\text { Lavage } \\
\text { and } \\
\text { closed }\end{array}$} & \multirow[b]{2}{*}{$\begin{array}{l}\text { Hemicol- } \\
\text { ectomy }\end{array}$} & \multirow[b]{2}{*}{$\begin{array}{l}\text { Colo- } \\
\text { stomy }\end{array}$} & \multirow[b]{2}{*}{$\begin{array}{l}\text { Modified } \\
\text { Grahams }\end{array}$} & \multirow[b]{2}{*}{$\begin{array}{l}\text { Nephre- } \\
\text { ctomy }\end{array}$} & \multirow[b]{2}{*}{ Biopsy } & \multirow[b]{2}{*}{$\begin{array}{l}\text { By-pass } \\
\text { surgery }\end{array}$} & \multirow[b]{2}{*}{ DOT } & \multirow[b]{2}{*}{$\begin{array}{l}\text { Splene- } \\
\text { ctomy }\end{array}$} & \multirow[b]{2}{*}{$\begin{array}{l}\text { Drainage } \\
\text { and lavage }\end{array}$} & \multirow[b]{2}{*}{$\begin{array}{l}\text { FB re- } \\
\text { moval }\end{array}$} & \multirow[b]{2}{*}{ Total } \\
\hline & $\begin{array}{l}\text { lleos- } \\
\text { tomy }\end{array}$ & $\begin{array}{c}\text { Resection } \\
\text { anasto- } \\
\text { mosis }\end{array}$ & & & & & & & & & & & & & & & \\
\hline Adhesions & $\begin{array}{c}1 \\
(12.5 \%)\end{array}$ & $2(25 \%)$ & $5(62.5 \%)$ & 0 & 0 & 0 & 0 & 0 & 0 & 0 & 0 & 0 & 0 & 0 & 0 & 0 & 8 \\
\hline $\begin{array}{l}\text { Perforated } \\
\text { ileum }\end{array}$ & $\begin{array}{c}4 \\
(57.1 \%)\end{array}$ & $2(28.6 \%$ & 0 & 0 & 0 & 0 & 0 & 0 & 0 & 0 & 0 & $1(14.3 \%)$ & 0 & 0 & 0 & 0 & 7 \\
\hline $\begin{array}{l}\text { Perforated } \\
\text { PUD }\end{array}$ & 0 & 0 & 0 & 0 & 0 & 0 & 0 & 0 & $4(100 \%)$ & 0 & 0 & 0 & 0 & 0 & 0 & 0 & 4 \\
\hline $\begin{array}{c}\text { Normal } \\
\text { abdomen }\end{array}$ & 0 & 0 & 0 & 0 & $1(20 \%)$ & $4(80 \%)$ & 0 & 0 & 0 & 0 & 0 & 0 & 0 & 0 & 0 & 0 & 5 \\
\hline Appenditis & 0 & 0 & 0 & 0 & $5(100 \%)$ & 0 & 0 & 0 & 0 & 0 & 0 & 0 & 0 & 0 & 0 & 0 & 5 \\
\hline $\begin{array}{l}\text { Intestinal } \\
\text { Gangrene }\end{array}$ & 0 & 3 (75\%) & 0 & 0 & 0 & 0 & 0 & 0 & 0 & 0 & 0 & 0 & $\begin{array}{c}1 \\
(25 \%)\end{array}$ & 0 & 0 & 0 & 4 \\
\hline $\begin{array}{l}\text { Compound } \\
\text { volvulus }\end{array}$ & 0 & $1(33.3 \%)$ & 0 & 0 & 0 & 0 & 0 & $\begin{array}{c}2 \\
(66.7 \%)\end{array}$ & 0 & 0 & 0 & 0 & 0 & 0 & 0 & 0 & 3 \\
\hline $\begin{array}{c}\text { Liver } \\
\text { Laceration }\end{array}$ & 0 & 0 & 0 & $1(50 \%)$ & 0 & $1(50 \%)$ & 0 & 0 & 0 & 0 & 0 & 0 & 0 & 0 & 0 & 0 & 2 \\
\hline $\begin{array}{l}\text { Perforated } \\
\text { Stomach }\end{array}$ & 0 & 0 & 0 & $1(50 \%)$ & 0 & 0 & 0 & 0 & $1(50 \%)$ & 0 & 0 & 0 & 0 & 0 & 0 & 0 & 2 \\
\hline $\begin{array}{l}\text { Ileo-ileal } \\
\text { knotting }\end{array}$ & $1(50 \%)$ & & $1(50 \%)$ & 0 & 0 & 0 & 0 & 0 & 0 & 0 & 0 & 0 & 0 & 0 & 0 & 0 & 2 \\
\hline $\begin{array}{l}\text { Terminal } \\
\text { ileitis }\end{array}$ & 0 & 0 & 0 & 0 & $1(50 \%)$ & $1(50 \%)$ & 0 & 0 & 0 & 0 & 0 & 0 & 0 & 0 & 0 & 0 & 2 \\
\hline $\begin{array}{l}\text { Intuss- } \\
\text { uception }\end{array}$ & 0 & $2(100 \%)$ & 0 & 0 & 0 & 0 & 0 & 0 & 0 & 0 & 0 & 0 & 0 & 0 & 0 & 0 & 2 \\
\hline $\begin{array}{l}\text { Sigmoid } \\
\text { Volulus }\end{array}$ & 0 & $1(50 \%)$ & 0 & 0 & 0 & 0 & 0 & $1(50 \%)$ & 0 & 0 & 0 & 0 & 0 & 0 & 0 & 0 & 2 \\
\hline Cancer colon & 0 & 0 & 0 & 0 & 0 & 0 & $1(100 \%)$ & 0 & 0 & 0 & 0 & 0 & 0 & 0 & 0 & 0 & 1 \\
\hline $\begin{array}{l}\text { Leaking } \\
\text { Anast- } \\
\text { omosis }\end{array}$ & 0 & 0 & 0 & 0 & 0 & 0 & 0 & $1(100 \%)$ & 0 & 0 & 0 & 0 & 0 & 0 & 0 & 0 & 1 \\
\hline $\begin{array}{c}\text { Redundant } \\
\text { Sigmoid colon }\end{array}$ & 0 & $1(100 \%)$ & 0 & 0 & 0 & 0 & 0 & 0 & 0 & 0 & 0 & 0 & 0 & 0 & 0 & 0 & 1 \\
\hline $\begin{array}{l}\text { Perforated } \\
\text { jujenum }\end{array}$ & 0 & $1(100 \%)$ & 0 & 0 & 0 & 0 & 0 & 0 & 0 & 0 & 0 & 0 & 0 & 0 & 0 & 0 & 1 \\
\hline $\begin{array}{l}\text { Perforated } \\
\text { Duodenum }\end{array}$ & 0 & 0 & 0 & $\begin{array}{c}1 \\
(100 \%)\end{array}$ & 0 & 0 & 0 & 0 & 0 & 0 & 0 & 0 & 0 & 0 & 0 & 0 & 1 \\
\hline $\begin{array}{l}\text { Ruptured } \\
\text { Kidney }\end{array}$ & 0 & 0 & 0 & 0 & 0 & 0 & 0 & 0 & 0 & $1(100 \%)$ & 0 & 0 & 0 & 0 & 0 & 0 & 1 \\
\hline Cecal mass & 0 & 0 & 0 & 0 & 0 & 0 & 0 & 0 & 0 & 0 & $\begin{array}{c}1 \\
(100 \%)\end{array}$ & 0 & 0 & 0 & 0 & 0 & 1 \\
\hline $\begin{array}{c}\text { Cancer } \\
\text { Pancreas }\end{array}$ & 0 & 0 & 0 & 0 & 0 & 0 & 0 & 0 & 0 & 0 & 0 & $1(100 \%)$ & 0 & 0 & 0 & 0 & 1 \\
\hline $\begin{array}{l}\text { Ruputred } \\
\text { Spleen }\end{array}$ & 0 & 0 & 0 & 0 & 0 & 0 & 0 & 0 & 0 & 0 & 0 & 0 & 0 & $1(100 \%)$ & 0 & 0 & 1 \\
\hline $\begin{array}{c}\text { Messenteric } \\
\text { Adenitis }\end{array}$ & 0 & 0 & 0 & 0 & 0 & $1(100 \%)$ & 0 & 0 & 0 & 0 & 0 & 0 & 0 & 0 & 0 & 0 & 1 \\
\hline $\begin{array}{l}\text { Perforated } \\
\text { Cecum }\end{array}$ & 0 & 0 & 0 & $\begin{array}{c}2 \\
(100 \%)\end{array}$ & 0 & 0 & 0 & 0 & 0 & 0 & 0 & 0 & 0 & 0 & 0 & 0 & 2 \\
\hline $\begin{array}{l}\text { Abdominal } \\
\text { Abscess }\end{array}$ & 0 & 0 & 0 & 0 & 0 & 0 & 0 & 0 & 0 & 0 & 0 & 0 & 0 & 0 & $1(100 \%)$ & 0 & 1 \\
\hline FB Stomach & 0 & 0 & 0 & 0 & 0 & 0 & 0 & 0 & 0 & 0 & 0 & 0 & 0 & 0 & 0 & $\begin{array}{c}1 \\
(100 \%)\end{array}$ & 1 \\
\hline $\begin{array}{l}\text { Perforated } \\
\text { Appendix }\end{array}$ & 0 & 0 & 0 & 0 & $2(100 \%)$ & 0 & 0 & 0 & 0 & 0 & 0 & 0 & 0 & 0 & 0 & 0 & 2 \\
\hline Total & $6(9.4 \%)$ & $13(20.3 \%)$ & $6(9.4 \%)$ & $5(7.8 \%)$ & $9(14.1 \%)$ & $\begin{array}{c}7 \\
(10.9 \%)\end{array}$ & $1(1.6 \%)$ & $4(6.3 \%)$ & $5(7.8 \%)$ & $1(1.6 \%)$ & $\begin{array}{c}1 \\
(1.6 \%)\end{array}$ & $2(3.1 \%)$ & $\begin{array}{c}1 \\
(1.6 \%)\end{array}$ & $1(1.6 \%)$ & $1(1.6 \%)$ & $\begin{array}{c}1 \\
(1.6 \%)\end{array}$ & $\begin{array}{c}64 \\
(100 \%)\end{array}$ \\
\hline
\end{tabular}

but resection and anastomosis was done in $25 \%$ of adhesion cases and ileostomy accounted $12.5 \%$ due to inadvertent ileal injury. In patients with ileal perforation $(n=7)$ the majority were treated with temporary loop or double end ileostomy
(57.1\%), resection-anastomosis was done in $28.6 \%$, ileotransverse bypass in $14.3 \%$ of cases.

However in all patients who had appendicitis $(n=5)$, conventional appendectomy was done. Furthermore there were 
5 cases of negative laparotomy findings, of which $80 \%$ were larvaged and closed and $20 \%$ had prophylactic appendectomy. Patients in whom small intestinal gangrene was found resection anastomosis was performed in $75 \%$ of the cases and $25 \%$ had a DOT (death on Table). These differences when compared was found to be statistically significant (Chi-square 378.864 , df 390, P value 0.000001). Therefore, a significantly proportion of cases of ileal perforation were treated with temporary ileostomy as well as adhesion with adhesiolysis, appendicitis with appendectomy, intestinal gangrene with resection anastomosis and negative laparotomy with larvage and closure.

Outcome of laparotomy; A variety of outcomes were recorded during the 30 days follow-up of patients after laparotomy and matched for the intra-operative (actual diagnosis, Table 4). As shown in Table 4, out of the 64 patients studied, the most frequent complication observed was surgical site infection (SSI) $(20 \%, \mathrm{n}=13)$, followed by wound dehiscence $(17.2 \%$, $\mathrm{n}=11)$, crude mortality rate was $15.6 \%,(\mathrm{n}=10)$ and complication requiring emergency re-operation $(10.9 \%, n=7)$. Furthermore the surgical condition with most prevalent complication was ileal perforation, out of the seven, 38.5\% developed SSI, wound dehiscence accounted for $27.3 \%$ and 2 died. Indeed amongst the 10 patients who died $2(20 \%, n=10)$ had perforated ileum. Postoperative peritonitis tended to be more common in patients with intestinal gangrene $(33.3 \%)$ of which $14.3 \%$ needed reoperation.

Also compared to rest of the surgical conditions, patients with ileal perforation were the most common groups whose duration of hospitalization was greater than 2weeks (25\%). Whereas all the above differences were not statistically significant ( $\mathrm{P}$ value $>$ 0.05), outcome of laparotomy such as wound dehiscence, SSI, and tendency to develop peritonitis and anastomotic leak were statistically significant ( $\mathrm{P}$ value $\leq 0.05$ ) when disaggregated within the different age groups. Therefore age is a significant factor in outcome of laparotomy.

\section{Discussion}

Despite low income countries having major burdens of the surgical diseases, one of commonest procedure - laparotomy, has been found to have a high mortality rate in many SubSaharan African countries [11]. Depending on patient-related, disease-related and intervention-related factors, surgical patients have different outcomes of which some recover uneventfully, some get complications which cause debilitation and others succumb to these morbidities $[12,13]$. In this study pre, intra and post laparotomy 30 days follow up was done to discern the temporal outcome of this common procedure in our setting.

In a study by Abebe, et al. [14], the mean age of laparotomy patients were found to be 29 years representing the youth and this is close to what was found in this study. Other researcher have reported a higher average ages for laparotomy patients with developed nations tending to have older patients $[15,16]$. Although the male: female ratio for laparotomy patients reported by Abebe, et al. (2019) is 6.2:1 [14], Khalilur, et al.
(2018) reported Male: Female ratio of 2.5:1 [17] and Lebowa, et al. [18], found a female: male ratio of 1.3:1 [18]. This study found Male: Female ratio of 1.5:1 close to that of Lebowa, et al. [18]. It appears therefore that laparotomy tend to have no particular gender related predilection.

In the study there was a positive correlation between clinical examination and the intra-operative findings $(\mathrm{r}=$ 0.405, P value $=0.001)$. Mir-Zeeshan, and Vamsee- Krishna (2019) also found that there is a 95\% accuracy rate of clinical diagnosis when compared to intra op diagnosis, Kappa is 0.912 $(p<0.0001)$ [19]. Therefore clinical judgment is key to diagnosis of acute abdomen and investigations are only supplementary. Regarding intra operative procedures, approximately $8 \%$, $(\mathrm{n}=5, \mathrm{~N}=64)$ had negative laparotomy a figure which is close the $5 \%$ rate reported by Abebe, et al. (2019). However in our setting clinical diagnosis and outcome of the intervention may be influenced by delay in presentation amongst others which include use of medications, premorbid conditions, and multiple visits to clinics prior to reaching a hospital where surgical services are offered.

Whereas appendicitis, intestinal adhesions, gangrene, peptic ulcer perforation and the other conditions were treated in a conventional way, ileal perforation was special (Table 3). In this study, ileostomy was preferred in $57.1 \%$, resection and primary anastomosis was done in $28.6 \%$, ileo-transverse bypass in $14.3 \%$ of cases of ileal perforation (Chi-square 378.864 , d.f 390, P value 0.000001). Previously another study also recommended that in ileal perforation, ileostomy may be given priority over other surgical options since post-operative complication rate is less (17.85\%) compared to $32.14 \%$ in cases of primary closure [17]

During the 30 days longitudinal follow-up of the post laparotomy patients, wound dehiscence rate was found to be $17.2 \%$. This finding is within range since rates of wound dehiscence following laparatomy have was previously found range from $0 \%$ to $44 \%$ depending on the wound type with contaminated/dirty wound having the highest rate of wound dehiscence [20]. Other authors have reported lower rates of wound dehiscence as low as $2.9 \%$ [21] and $5.1 \%$ [22] respectively but all aver that the rate of wound dehiscence depend on status of the wound. Indeed Surgical site infection (SSI) is a risk factor for developing wound dehiscence [21] This study also found surgical site infection (SSI) rate of $20 \%$. Other researchers have found similar SSI rates of $16.4 \%$ [24] and $23.2 \%$ [25] respectively. Furthermore, immunosuppressive medications, open cholecystectomy, and dirty wound have been found to be significantly associated with SSI [23]. In 2014, Ramneesh, , et al. reported that the risk of wound dehiscence is more common in emergency laparotomy and $88 \%$ of patients with contaminated or dirty wounds tend to develop wound dehiscence.

The crude mortality rate of $15.6 \%$ reported in this study is similar the mortality rate of $16.7 \%$, reported by Hietbrink, et al. [7] and is within post-laparatomy mortality range of $10.5-21 \%$ previously reported Anwar, et al. [15] and 9.6-33\% by Howes, et al. (2015) [26]. Baison [11] found the post-operative mortality 
Table 4: The Outcome of Laparotomy - Editable.

\begin{tabular}{|c|c|c|c|c|c|c|c|c|}
\hline & \multicolumn{7}{|c|}{ 30Days Outcome } & \multirow[b]{2}{*}{$\begin{array}{c}\text { Hospitalized more than } \\
\text { 2weeks }\end{array}$} \\
\hline Intra-op Findings & $\begin{array}{c}\text { Wound } \\
\text { dehiscence }\end{array}$ & $\begin{array}{l}\text { Surgical site } \\
\text { Infection }\end{array}$ & Peritonitis & $\begin{array}{c}\text { Anastomotic } \\
\text { Leak }\end{array}$ & Re-operated & Organ failure & Died & \\
\hline Perforated ileum $(n=7)$ & $3(27.3 \%)$ & $5(38.5)$ & $1(16.7 \% \%$ & $1(25 \%)$ & $2(28.6 \%)$ & $1(20.0 \%)$ & $2(20 \%)$ & $2(25 \%)$ \\
\hline Perforated PUD $(n=4)$ & $2(18.2 \%)$ & $1(7.70 \%)$ & $1(16.7 \% \%$ & $1(25 \%)$ & $2(28.6 \%)$ & 0 & 0 & $1(12.5 \%)$ \\
\hline Perforated jujenum $(n=1)$ & 0 & 0 & 0 & 0 & 0 & 0 & 0 & 0 \\
\hline Liver Laceration $(n=2)$ & $1(9.1 \%)$ & 0 & 0 & 0 & 0 & $1(20.0 \%)$ & $1(10 \%)$ & $1(12.5 \%)$ \\
\hline Appenditis $(n=5)$ & 0 & $1(7.70 \%)$ & $1(16.7 \% \%$ & 0 & 0 & $1(20.0 \%)$ & $1(10 \%)$ & 0 \\
\hline Intestinal Gangrene $(n=4)$ & $1(9.1 \%)$ & $2(15.4 \%)$ & $2(33.3 \%)$ & $1(25 \%)$ & $1(14.3 \%)$ & $1(20.0 \%)$ & $2(20 \%)$ & $1(12.5 \%)$ \\
\hline Normal abdomen $(n=5)$ & 0 & 0 & 0 & 0 & 0 & 0 & 0 & 0 \\
\hline Cancer colon $(n=1)$ & 0 & $1(7.70 \%)$ & $1(16.7 \%)$ & $1(25 \%)$ & $1(14.3 \%)$ & 0 & $1(10 \%)$ & $1(12.5 \%)$ \\
\hline Compound volvulus $(n=3)$ & $1(9.1 \%)$ & 0 & 0 & 0 & 0 & 0 & 0 & $1(12.5 \%)$ \\
\hline Leaking Anastomosis $(n=1)$ & $1(9.1 \%)$ & $1(7.70 \%)$ & 0 & 0 & 0 & 0 & 0 & \\
\hline $\begin{array}{l}\text { Redundant Sigmoid colon } \\
\qquad(n=1)\end{array}$ & 0 & 0 & 0 & 0 & 0 & 0 & 0 & 0 \\
\hline Adhesions $(n=8)$ & $1(9.1 \%)$ & 0 & 0 & 0 & $1(14.3 \%)$ & 0 & 0 & 0 \\
\hline Perforated Stomach $(n=2)$ & 0 & 0 & 0 & 0 & 0 & 0 & 0 & 0 \\
\hline Ileo-ileal knotting $(n=2)$ & 0 & 0 & 0 & 0 & 0 & 0 & 0 & 0 \\
\hline Terminal ileitis $(n=2)$ & 0 & 0 & 0 & 0 & 0 & 0 & 0 & 0 \\
\hline Ruptured Kidney $(n=1)$ & 0 & 0 & 0 & 0 & 0 & 0 & 0 & \\
\hline Sigmoid Volulus $(n=2)$ & 0 & 0 & 0 & 0 & 0 & 0 & 0 & 0 \\
\hline Perforated Duodenum $(n=1)$ & 0 & 0 & 0 & 0 & 0 & 0 & 0 & 0 \\
\hline Intussuception ( $n=2)$ & $1(9.1 \%)$ & $2(15.4 \%)$ & 0 & 0 & 0 & 0 & 0 & $1(12.5 \%)$ \\
\hline Cecal mass $(n=1)$ & 0 & 0 & 0 & 0 & 0 & 0 & 0 & 0 \\
\hline Cancer Pancreas $(n=1)$ & 0 & 0 & 0 & 0 & 0 & 0 & 0 & 0 \\
\hline Ruputred Spleen $(n=1)$ & 0 & 0 & 0 & 0 & 0 & 0 & 0 & 0 \\
\hline Messenteric Adenitis $(n=1)$ & 0 & 0 & 0 & 0 & 0 & 0 & 0 & 0 \\
\hline Perforated Cecum $(n=2)$ & 0 & 0 & 0 & 0 & 0 & 0 & $1(10 \%)$ & 0 \\
\hline Abdominal Abscess $(n=1)$ & 0 & 0 & 0 & 0 & 0 & 0 & $1(10 \%)$ & \\
\hline FB Stomach $(n=1)$ & 0 & 0 & 0 & 0 & 0 & 0 & 0 & 0 \\
\hline Perforated Appendix $(n=2)$ & 0 & 0 & 0 & 0 & 0 & $1(20.0 \%)$ & $1(10 \%)$ & 0 \\
\hline Total & $11(100 \%)$ & $13(100 \%)$ & $6(100 \%)$ & $4(100 \%)$ & $7(100 \%)$ & $5(100 \%)$ & $10(100 \%)$ & $8(100 \%)$ \\
\hline
\end{tabular}

rate in Rwanda to be $12 \%$ following laparotomy surgery. This study found unplanned emergency re-operation rate was $11 \%$. In Ethiopia, similar re-laparatomy rates $(12 \%)$ was found by Nurhusien, et al. [27]. Generally, ileal perforations tend to more ominous than all the other indications for laparotomy. Other researchers found ileal perforations to have higher mortality ranges from $11.5 \%$ to $50 \%$ [28]. Regarding ileal perforations early presentation and diagnosis, adequate resuscitation, prompt surgery and vigorous post-operative management improves mortality rates and complication rates are less when treated with ileostomy $[17,28]$. Furthermore as in this study, age was been found to significantly affect outcome of laparotomy as was similarly found by other researchers $[26,29,30]$.

\section{Conclusion}

Pre-operative clinical examination, investigation are important to determine the need for laparotomy since it positively correlates with the intra-operative findings $(r=$ 0.405 , P value $=0.001$ ). Whereas in 30days post laparotomy period wound complications tend predominate, ileal perforation need special attention because of the less favorable outcome especially where ileostomy is not prioritized. Age tend to significantly affect laparotomy outcome ( $\mathrm{P}$ value $\leq 0.05$ ).

\section{Foot note}

\section{Contribution of the authors}

1. Dr Amone Derrick is the principle investigator who developed the proctocol, corrected it until approval by the Research Ethics Committee (REC). He supervised the data collection, data entry and wrote the manuscript.

2. Dr Okello Tom Richard, is the co-principle investigator. He supervised the protocol development data entry, analysis and wrote the manuscript.

3. Dr Okot Christoper and Dr Kitara David Lagoro supervised the protocol development, presentation to the REC, proof read the manuscript 
4. Dr Mugabi Patrick Participated in protocol development, he also supervised the data analysis and writing of the result section of this manuscript

5. Dr Ogwang David Martin proof read the manuscript and particularly revised and improved the discussion part of the manuscript.

Declaration of conflict of interest: We the authors hereby aver that we do not have any conflict of interest neither have we received notice nor declaration of conflict of interest in this research project from inception, protocol development and approval as well as conducting study and writing the report and manuscript.

\section{Funding}

This work was supported through the DELTAS Africa Initiative Grant \# DEL-15-011 to THRiVE-2. The funders had no role in the study design, data collection, and analysis, decision to publish, or preparation of the manuscript.

\section{References}

1. Barrow E, Anderson ID, Varley S, Pichel AC, Peden CJ, , et al.. (2013) Current UK practice in emergency laparotomy. Ann R Coll Surg Engl 95: 599-603. Link: https://bit.ly/3kbmSd3

2. Strik C, Stommel MWJ, Schipper LJ, Van Goor H, Ten Broek RPG (2016) Risk factors for future repeat abdominal surgery. Langenbecks Arch Surg 401: 829. Link: https://bit.ly/3kcu9Jt

3. Vester-Andersen M, Lundstrøm LH, Møller MH, Waldau T, Rosenberg J, , et al.. (2014) Mortality and postoperative care pathways after emergency gastrointestinal surgery in 2904 patients: a population-based cohort study. $\mathrm{Br}$ J Anaes 112: 860-870. Link: https://bit.ly/2JQFJ0v

4. Gejoe G, Yadev I, Rahul M (2017) Emergency Laparotomies at a Tertiary Care Center-a Hospital-Based Cross-Sectional Study. Indian J Surg 79: 206-211. Link: https://bit.ly/3eDoATg

5. Kakande I, Ekwaro L, Obote WW, Nassali G, Kyamanywa P (2001) Intestinal volvulus at St Francis Hospital, Kampala. East Central AfrJ Surg 6. Link: https://bit.ly/38nLSLV

6. Ruhinda G, Kyamanywa P, Kitya D, Bajunirwe F (2008) Abdominal Injuries at Mbarara Regional Referral Hospital, Uganda. East Central Afr J Surg 13: 29-36. Link: https://bit.ly/3n655G1

7. Hietbrink $F$, Smeeing $D$, Karhof $S$, Jonkers HF, Houwert $M$, et al. (2019) Outcome of trauma-related emergency laparotomies, in an era of far-reaching specialization. World J Emerg Surg 14: 40. Link: https://bit.ly/38q2pic

8. Okeny PK, Hwang TG, Ogwang DM (2011) Acute Bowel Obstruction in a Rural Hospital in Northern in Northern Uganda. East Central Afr J Surg 12: 67-73. Link: https://bit.ly/2GG8OdK

9. Tengberg LT, Cihoric M, Foss NB, Bay-Nielsen M, , et al.. (2016) Complications after emergency laparotomy beyond the immediate postoperative period - a retrospective, observational cohort study of 1139 patients. Anaesthesia: 309 316. Link: https://bit.ly/317tEBP

10. Bansal AR, Mallick MR, Jena S (2019) A study of post-operative complications of all emergency laparotomy in a tertiary care hospital within 90 days. Archives of Clinical Gastroenterology 15-18. Link: https://bit.ly/38tcV8v

11. Baison GN (2015) Outcomes of Laparotomy at a Large Referral Center in Rwanda. Link: https://bit.ly/3kgh69Y
12. Nuhu A, Bata MG (2010) Causes and treatment outcome of perforation peritonitis in north eastern Nigeria. Surg Pract 14: 92-96. Link: https://bit.ly/3kbXmEv

13. Testini M, Portincasa P, Piccini G, Lissidini G, Pellegrini F, et al.. (2003) Significant factors associated with fatal outcome in emergency open surgery for perforated peptic ulcer. World J Gastroenterol 9: 2338-2340. Link: https://bit.ly/3eDonzm

14. Abebe K, Bekele M, Tsehaye A, Lemmu B, Abebe E (2019) Laparotomy for Abdominal Injury Indication \& Outcome of patients at a Teaching Hospital in Addis Ababa, Ethiopia. Ethiop J Health Sci 29: 503-512. Link: https://bit.ly/2llo3d1

15. Anwar H, Fahad M, Chui T, Sadaf J, David L, , et al.. (2017) Patient outcome of emergency laparotomy improved with increasing "number of surgeons oncall" in a university hospital: Audit loop. Ann Med Surg (Lond) 23: 21-24. Link: https://bit.ly/3mXShlO

16. Fowler AJ, Abbott TEF, Prowle J, Pearse RM (2019) Age of patients undergoing surgery. Link: https://bit.ly/3IpUpBP

17. Rahman KA, Krishnaswamy J, Muthukumaran G, Sanjay Prakash JA (2018) comparative study on outcome of ileal perforation after primary perforation closure and resection and ileostomy. Int Surg J 5: 445-451. Link: https://bit.ly/38IDU61

18. Lebowa W, Skorus U, Rapacz K (2020) Indications for Emergency Abdomina Surgeries in Older Patients: 7-Year Experience of a Single Centre. Indian J Surg. Link: https://bit.ly/3n6B8Ws

19. Mir Zeeshan A, Vamsee Krishna M (2019) Comparative analysis of clinical, radiological and operative findings in acute abdomen. Int Surg J 6: 806-811. Link: https://bit.ly/32tVQYq

20. Bates C (2018) World Union of Wound Healing Societies (WUWHS) Consensus Document. Surgical wound dehiscence: improving prevention and outcomes. Wounds International, 2018, Omnia-Med Ltd 1.01 Cargo Works, 1-2 Hatfields, London, SE1 9PG

21. Jakub K, Piotr R, Sabina Ż, Lasek A, Zbierska K (2012) Risk factors for wound dehiscence after laparotomy - clinical control trial, Pol Przegl Chir 84: 565-573. Link: https://bit.ly/3n3Lc2i

22. Helgeland J, Tomic O, Hansen TM, Kristoffersen DT, Hassani S, et al.. (2019) Postoperative wound dehiscence after laparotomy: a useful healthcare quality indicator? A cohort study based on Norwegian hospital administrative data. BMJ Open 9: e026422. Link: https://bit.ly/3kgvm2l

23. Emil A, Keinan-Boker L, Eithan A, Mais T, Rabinovich A, et al.. (2015) Surgical site infections after abdominal surgery: incidence and risk factors. A prospective cohort study Infect Dis (Lond) 47: 761-767. Link: https://bit.ly/2JLSeKF

24. Lubega A, Bazira J, Najjuka JL (2017) Incidence and Etiology of Surgical Site Infections among Emergency Postoperative Patients in Mbarara Regional Referral Hospital, South Western Uganda. Link: https://bit.ly/32qKnsG

25. Harrison EW (2018) Surgical site infection after gastrointestinal surgery in high-income, middle-income, and low-income countries: a prospective international, multicentre cohort study. Lancet Infect Dis 18: 516-525. Link: https://bit.ly/35dDYCV

26. Howes TE, Cook TM, Corrigan LJ, Dalton SJ, Richards SK, , et al. (2015) Postoperative morbidity survey, mortality and length of stay following emergency laparotomy. Anaesthesia 70: 1020-1027. Link: https://bit.ly/3806TG9

27. Nurhusien NY, Yeneabat BY, Debrework TB, Walle TA, Netsere HB, et al.. (2019) Prevalence and factors associated with re-laparotomy among patients operated in Debre-Markos Referral Hospital, North West Ethiopia: Retrospective cross- sectional study.s General Surgery. Link: https://bit.ly/32tWPb4

Citation: Amone D, Okello TR, Okot C, Kitara DL, Mugabi P, et al. (2020) Short-term outcomes of laparotomy in the two teaching hospitals of gulu university, northern uganda. Arch Clin Gastroenterol 6(3): 069-076. DOI: https://dx.doi.org/10.17352/2455-2283.000084 
28. Ugochukwu Al, Amu OC, Nzegwu MA (2013) Ileal perforation due to typhoid fever - Review of operative management and outcome in an urban centre in Nigeria. Int J Surg 11: 218-222. Link: https://bit.ly/3n6JUU6

29. Mačiulienè A, Maleckas A, Kriščiukaitis A, Mačiulis VJ, Macas A, , et al.. (2019) Predictors of 30-Day In-Hospital Mortality in Patients Undergoing Urgent Abdominal Surgery Due to Acute Peritonitis Complicated with Sepsis. Med Sci Monit 25: 6331-6340. Link: https://bit.ly/3kbsl3E
30. Nader NM, Victor JL, Symons RG, McCormick WC, Flum DR (2009) Impact of Advancing Age on Abdominal Surgical Outcomes. Arch Surg 144: 1108-1114. Link: https://bit.ly/358ngVj

31. Ramneesh G, Sheerin S, Surinder S, Bir S (2014) A Prospective Study of Predictors for Post Laparotomy Abdominal Wound Dehiscence, J Clin Diagn Res 8: 80-83. Link: https://bit.ly/3n7fWzy

\section{Discover a bigger Impact and Visibility of your article publication with}

\section{Peertechz Publications}

\section{Highlights}

* Signatory publisher of ORCID

- Signatory Publisher of DORA (San Francisco Declaration on Research Assessment)

* Articles archived in worlds' renowned service providers such as Portico, CNKI, AGRIS, TDNet, Base (Bielefeld University Library), CrossRef, Scilit, J-Gate etc.

* Journals indexed in ICMJE, SHERPA/ROMEO, Google Scholar etc.

- OAI-PMH (Open Archives Initiative Protocol for Metadata Harvesting)

* Dedicated Editorial Board for every journal

* Accurate and rapid peer-review process

* Increased citations of published articles through promotions

* Reduced timeline for article publication

Submit your articles and experience a new surge in publication services (https://www.peertechz.com/submission).

Peertechz journals wishes everlasting success in your every endeavours.

Copyright: @ 2020 Amone D, et al. This is an open-access article distributed under the terms of the Creative Commons Attribution License, which permits unrestricted use distribution, and r eproduction in any medium, provided the original author and source are credited.

Citation: Amone D, Okello TR, Okot C, Kitara DL, Mugabi P, et al. (2020) Short-term outcomes of laparotomy in the two teaching hospitals of gulu university, northern uganda. Arch Clin Gastroenterol 6(3): 069-076. DOI: https://dx.doi.org/10.17352/2455-2283.000084 